\title{
SOME EXAMPLES OF MINIMALLY DEGENERATE MORSE FUNCTIONS
}

\author{
by FRANCES KIRWAN
}

(Received 23rd October 1985)

Let $X$ be a compact Riemannian manifold. If $f: X \rightarrow \mathbb{R}$ is a nondegenerate Morse function in the sense of Bott [2] then one has Morse inequalities which can be expressed in the form

$$
P_{\imath}(X)=\sum_{\beta \in B} t^{\lambda(\beta)} P_{t}\left(C_{\beta}\right)-(1+t) Q(t), \quad Q(t) \geqq 0
$$

where $P_{t}(X)$ is the Poincare polynomial $\sum t^{i} \operatorname{dim} H^{i}(X ; \mathbb{Q})$ of $X$ and $\left\{C_{\beta} \mid \beta \in B\right\}$ are the connected components of the set of critical points for $f$. For any polynomial $Q(t) \in \mathbb{Z}[t]$ we write $Q(t) \geqq 0$ if all the coefficients of $Q$ are nonnegative.

The purpose of this note is to give some examples of functions $f$ which are not nondegenerate (indeed the components of the set of cricitcal points may have serious singularities) but nonetheless satisfy the Morse inequalities. The basic idea can be found in [3] where it is applied to one particular function.

One says that a smooth function $f: X \rightarrow \mathbb{R}$ is minimally degenerate if the set of its critical points $\operatorname{Crit}(f)$ is a finite disjoint union of closed subsets $\left\{C_{\beta} \mid \beta \in B\right\}$ of $X$, along each of which there exists a minimising manifold for $f$ in the following sense. A locally closed submanifold $\Sigma_{\beta}$ of $X$ with orientable normal bundle, which contains $C_{\beta}$ and is closed in a neighbourhood of $C_{\beta}$, is a minimising manifold for $f$ along $C_{\beta}$ if

(i) the restriction of $f$ to $\Sigma_{\beta}$ achieves its minimum value exactly on $C_{\beta}$; and

(ii) the tangent space to $\Sigma_{\beta}$ at any $x \in C_{\beta}$ is maximal among those subspaces of $T_{x} X$ on which the Hessian $H_{x}(f)$ of $f$ at $x$ is nonnegative semi-definite.

We may assume without loss of generality that each $\Sigma_{\beta}$ is connected and hence may define the index of $f$ along $C_{\beta}$ as

$$
\lambda(\beta)=\operatorname{codim} \Sigma_{\beta}
$$

Theorem ([3] Theorem 10.2). Let $f: X \rightarrow \mathbb{R}$ be a minimally degenerate Morse function with critical subsets $\left\{C_{\beta} \mid \beta \in B\right\}$. Then the Morse inequalities

$$
P_{t}(X)=\sum_{\beta \in B} t^{\lambda(\beta)} P_{t}\left(C_{\beta}\right)-(1+t) Q(t), \quad Q(t) \geqq 0
$$

are satisfied. 
Remark. Here the Poincaré polynomial is defined using Čech cohomology.

Any nondegenerate Morse function is minimally degenerate. This note gives a method of constructing examples of minimally degenerate Morse functions which are not nondegenerate by using convex functions of nondegenerate Morse functions.

Suppose that $f_{1}, \ldots, f_{n}$ are real-valued functions on $X$ such that their gradient vector fields $\operatorname{grad} f_{1}, \ldots, \operatorname{grad} f_{n}$ commute. (In fact it suffices to assume that each critical set $\operatorname{Crit}\left(f_{j}\right)$ is invariant under the gradient flow $\operatorname{grad} f_{k}$ for $k \neq j$.) Let

$$
c: \mathbb{R}^{n} \rightarrow \mathbb{R}
$$

be a strictly convex smooth function on $\mathbb{R}^{n}$, and define $f: X \rightarrow \mathbb{R}$ by

$$
f=c\left(f_{1}, \ldots, f_{n}\right)
$$

Theorem. Suppose that $\sum_{i \leqq j \leqq n} \lambda_{j} f_{j}$ is a nondegenerate Morse function on $X$ for every $\left(\lambda_{1}, \ldots, \lambda_{n}\right) \in \mathbb{R}^{n}$. Then $f$ is a minimally degenerate Morse function.

In order to prove this we must first describe the set Crit $(f)$ of critical points for $f$. Since for each $j$ the critical set $\operatorname{Crit}\left(f_{j}\right)$ is a finite disjoint union of submanifolds of $X$ on each of which $f_{j}$ is constant, the image under

$$
F=\left(f_{1}, \ldots, f_{n}\right): X \rightarrow \mathbb{R}^{n}
$$

of the intersection

$$
\bigcap_{i \leqq j \leqq n} \operatorname{Crit}\left(f_{j}\right)
$$

is a finite set of points $A=\left\{\alpha_{1}, \ldots, \alpha_{m}\right\}$ in $\mathbb{R}^{n}$.

Lemma A. The image of $F: X \rightarrow \mathbb{R}^{n}$ is contained in the convex hull Conv $A$ of $A$ in $\mathbb{R}^{n}$.

Proof. It is enough to show that for every $\left(\lambda_{1}, \ldots, \lambda_{n}\right) \in \mathbb{R}^{n}$ the restriction to $F(X)$ of the linear functional

$$
y \rightarrow \sum_{1 \leqq i \leqq n} \lambda_{j} y_{j}
$$

on $\mathbb{R}^{n}$ takes its maximum value at some $\alpha_{i} \in A_{j}$; or alternatively that the function $\sum \lambda_{i} f_{j}$ on $X$ takes its maximum value at some $x \in \bigcap$ Crit $\left(f_{j}\right)$. This follows immediately from the hypothesis that $\operatorname{grad} f_{1}, \ldots, \operatorname{grad} f_{n}$ commute.

For each nonempty subset $A^{1}$ of $A$ the restriction of $c: \mathbb{R}^{n} \rightarrow \mathbb{R}$ to the convex hull Conv $A^{1}$ of $A^{1}$ takes its minimum value at a unique point $\beta$. Let $B$ be the set of all such points $\beta$ in $\mathbb{R}^{n}$. For each $\beta \in B$ let

$$
\lambda_{j}^{\beta}=\frac{\partial c}{\partial x_{j}}(\beta)
$$


for $1 \leqq j \leqq n$ and let $f_{\beta}=\sum \lambda_{j}^{\beta} f_{j}$. Let

$$
C_{\beta}=F^{-1}(\beta) \cap \operatorname{Crit}\left(f_{\beta}\right)
$$

Lemma B. The set of critical points $\operatorname{Crit}(f)$ for $f$ is the disjoint of the closed subsets $\left\{C_{\beta} \mid \beta \in B\right\}$ of $X$.

Proof. The subsets $\left\{C_{\beta} \mid \beta \in B\right\}$ are clearly closed and disjoint.

Suppose that $\operatorname{grad} f(x)=0$ and let $\beta=F(x)$. Then

$$
0=\sum_{1 \leqq j \leqq n} \frac{\partial c}{\partial x_{j}}(\beta) \operatorname{grad} f_{j}(x)=\operatorname{grad} f_{\beta}(x)
$$

so $x \in \operatorname{Crit}\left(f_{\beta}\right)$. Therefore it remains only to show that $\beta \in B$.

Let $S$ be the connected component of $\operatorname{Crit}\left(f_{\beta}\right)$ which contains $x$. By assumption $f_{\beta}$ is nondegenerate so $S$ is a submanifold of $X$. Moreover

$$
\left.\operatorname{grad} f_{j}\right|_{s}=\operatorname{grad}\left(\left.f_{j}\right|_{s}\right)
$$

for $1 \leqq j \leqq n$. Therefore if

$$
A^{1}=F\left(\bigcap_{1 \leqq j \leqq n} \operatorname{Crit}\left(f_{j}\right) \cap S\right)
$$

then $A^{1} \subseteq A$ and by Lemma $\mathrm{A}$

$$
\beta \in \operatorname{Conv} A^{1} \text {. }
$$

Let $\beta_{j}=f_{j}(x)$ for $1 \leqq j \leqq n$ so that $\beta=\left(\beta_{1}, \ldots, \beta_{n}\right)$. By the definition of $S$ its image under $F$ is contained in the hyperplane

$$
H=\left\{y \mid \sum \lambda_{j}^{\beta} y_{j}=\sum \lambda_{j}^{\beta} \beta_{j}\right\}
$$

of $\mathbb{R}^{n}$. Hence Conv $A^{1} \subseteq H$. Since $\lambda_{j}^{\beta}=\left(\partial c / \partial x_{j}\right)(\beta)$ the restriction of $c$ to $H$ has a critical point at $\beta$, which must be a minimum since $c$ is strictly convex. Therefore $\beta \in B$ and the lemma is proved.

Lemma C. For each $\beta \in B$ there exists a minimising manifold for $f$ along $C_{\beta}$.

Proof. Let $S$ be a connected component of $\operatorname{Crit}\left(f_{\beta}\right)$ which meets $F^{-1}(\beta)$. It is enough to find a minimising manifold for $f$ along $F^{-1}(\beta) \cap S$. Let $Y$ be the set of all $x \in X$ whose paths of steepest descent under $f_{\beta}$ converge to points of $S$. Since $f_{\beta}$ is nondegenerate $Y$ is a locally closed submanifold of $S$.

If $x \in Y$ then $\sum \lambda_{j}^{\beta} f_{j}(x) \geqq \sum \lambda_{j}^{\beta} \beta_{j}$. But the hyperplane

$$
H=\left\{y \mid \sum \lambda_{j}^{\beta} y_{j}=\sum \lambda_{j}^{\beta} \beta_{j}\right\}
$$


in $\mathbb{R}^{n}$ is a supporting hyperplane for the convex set $\{y \mid c(y) \leqq c(\beta)\}$. Thus we see that if $x \in Y$ then

$$
f(x)=c(F(x)) \geqq c(\beta)
$$

and equality holds if and only if $x \in S \cap F^{-1}(\beta)$.

Suppose that $x \in S \cap F^{-1}(\beta)$ and that $\xi$ is a vector in the orthogonal complement $T_{x} Y^{\perp}$ to $T_{x} Y$ in $T_{x} X$. Then

$$
d f_{j}(x)(\xi)=\operatorname{grad} f_{j}(x) \cdot \xi=0
$$

because $\operatorname{grad} f_{j}(x) \in T_{x} S \subseteq T_{x} Y$ for $1 \leqq j \leqq n$. Let Exp: $T X \rightarrow X$ be the exponential map. Then

$$
F(\operatorname{Exp} t \xi)=\beta+e(t)
$$

for $t \in \mathbb{R}$ where $e(t)=\left(e_{1}(t), \ldots, e_{n}(t)\right)$ is $O\left(t^{2}\right)$ as $t \rightarrow 0$. Hence

$$
f_{\beta}(\operatorname{Exp} t \xi)=\sum_{1 \leqq j \leqq n} \lambda_{j}^{\beta} \beta_{j}+\sum_{1 \leqq j \leqq n} \lambda_{j}^{\beta} e_{j}(t)
$$

and

$$
c(\operatorname{Exp} t \xi)=c(\beta)+\sum_{1 \leqq j \leqq n} \frac{\partial c}{\partial x_{j}}(\beta) e_{j}(t)+O\left(t^{3}\right)
$$

as $t \rightarrow 0$. It follows that the restriction to $T_{x} Y^{\perp}$ of the Hessian of $f$ at $x$ coincides with the restriction of the Hessian of $f_{\beta}$, which is negative definite. Thus we have proved that $Y$ is a minimising manifold for $f$ along $S \cap F^{-1}(\beta)$.

This completes the proof of the theorem.

Remarks (1). Notice that each critical set $C_{\beta}$ is the minimum set for the restriction of $f$ to a certain submanifold of $X$ which is invariant under the gradient flows of $f_{1}, \ldots, f_{n}$ (namely the union of those connected components of $\operatorname{Crit}\left(f_{\beta}\right)$ on which $f_{\beta}$ takes the value $\sum \lambda_{j}^{\beta} \beta_{j}$ ).

(2). Lemma $A$ is related to the paper [1] in which Atiyah shows that for certain functions $f_{1}, \ldots, f_{n}$ (those in Example (iii) below) one has

$$
F(X)=\text { Conv } A \text {. }
$$

Examples. (i). Let $n=1$ and let $c: \mathbb{R} \rightarrow \mathbb{R}$ be given by

$$
c(x)=(x-a)^{2}
$$

for some $a \in \mathbb{R}$. If $g: X \rightarrow \mathbb{R}$ is any nondegenerate Morse function with critical sets 
$\left\{C_{\beta} \mid \beta \in B\right\}$ then $f=(g-a)^{2}$ is minimally degenerate and one has inequalities of the form

$$
P_{t}\left(g^{-1}(a)\right)=P_{t}(X)-\sum_{\beta \in B^{1}} t^{\lambda(\beta)} P_{t}\left(C_{\beta}\right)+Q(t)(1+t), \quad Q(t) \geqq 0
$$

where $B^{1}=\left\{\beta \in B \mid g\left(C_{\beta}\right) \neq a\right\}$. Of course this can also be seen directly.

(ii) Let $X=X_{1} \times \cdots \times X_{n}$ and let $g_{j}: X_{j} \rightarrow \mathbb{R}$ be a nondegenerate Morse function on $X_{j}$ for $1 \leqq j \leqq n$. Then the function $f: X \rightarrow \mathbb{R}$ defined by

$$
f\left(x_{1}, \ldots, x_{n}\right)=c\left(g_{1}\left(x_{1}\right), \ldots, g_{n}\left(x_{n}\right)\right)
$$

is minimally degenerate for any strictly convex smooth $c: \mathbb{R}^{n} \rightarrow \mathbb{R}$, and Crit $(f)$ can be described as in Lemma B in terms of the critical sets for $g_{1}, \ldots, g_{n}$.

(iii) Suppose that $X$ is a symplectic manifold with symplectic form $\omega$ and that a compact torus $T=\left(S^{1}\right)^{n}$ acts on $X$ preserving $\omega$. Suppose that there exists a momentum map for this action, or equivalently that there exist functions $f_{1}, \ldots, f_{n}: X \rightarrow \mathbb{R}$ satisfying

$$
d f_{j}(x)(\xi)=\omega_{x}\left(\xi, a_{x}^{(D)}\right)
$$

for all $x \in X$ and $\xi \in T_{x} X$, where $a^{(1)}, \ldots, a^{(n)}$ is a basis for Lie $T$ and the vector field on $X$ induced by any $a \in \operatorname{Lie} T$ is denoted by $x \rightarrow a_{x}$. There exists a $T$-invariant almostcomplex structure $J$ on $X$ and a $T$-invariant Riemannian metric compatible with $\omega$ and $J$ such that

$$
\operatorname{grad} f_{j}(x)=J a_{x}^{(j)}
$$

for $1 \leqq j \leqq n$. Thus grad $f_{1}, \ldots, f_{n}$ commute and

$$
f=c\left(f_{1}, \ldots, f_{n}\right)
$$

is a minimally degenerate Morse function on $X$ for any strictly convex smooth $c: \mathbb{R}^{n} \rightarrow \mathbb{R}$.

This is the example studied in [3], where it is shown that the Morse inequalities with respect to equivariant cohomology are in fact equalities and lead to precise formulas for the Betti numbers of the symplectic quotient of $X$ by $T$.

\section{REFERENCES}

1. M. F. AтוYah, Convexity and commuting Hamiltonians, Bull. London Math. Soc. 14 (1982), $1-15$.

2. R. Botr, Nondegenerate critical manifolds, Ann. of Math. 60 (1954), 248-261.

3. F. C. KIRWAN, Cohomology of quotients in algebraic and symplectic geometry (Mathematical Notes 31, Princeton, 1985).

Mathematical Institute

St Giles

OXFORD 